A. Pramesh Rao, G. Swarup and Gopal-Krishna, eds.

\title{
Statistics of Giant Radio Sources
}

\author{
J. Machalski and M. Jamrozy
}

Jagellonian University, ul. Orla 171, 30-244 Kraków, Poland

\section{Introduction}

An important aspects of the studies of giant radio sources is that they can constrain models of the cosmological evolution of radio sources (e.g. Condon 1984, ApJ, 287, 461; Dunlop \& Peacock 1990, MNRAS, 247, 19) and their host galaxies, as well as the recent analytical models of time evolution of these sources (e.g. Kaiser, Dennett-Thorpe \& Alexander 1997, MNRAS, 292, 723; Blundell et al. 1999, AJ, 117, 677). In order to achieve these goals, we undertook the following tasks:

1) to derive the number-counts of giants with $D>1 \mathrm{Mpc}\left(q_{o}=0.5, H_{o}=50\right.$ $\left.\mathrm{km} \mathrm{s}^{-1} \mathrm{Mpc}^{-1}\right)$ as a function of their luminosity $(L)$ and redshift $(z)$, expected from the existing models of cosmological evolution,

2) to compare them with the observational data available, and

3 ) to determine the parameter space where there are no data points, and look for a possible cause of this bias.

In this contribution we present results of these tasks.

\section{Calculations}

In order to calculate the expected numbers of giants, we assumed the linear-size dependent Radio Luminosity Function (RLF) of the Fanaroff-Riley type II (FR II) sources in the form:

$$
\phi_{F R I I}(L, z, D)=\phi_{F R I I}(L, z) \lambda[D(L, z)],
$$

and derived:

(i) $\phi_{F R I I}(L, z)-1.4 \mathrm{GHz}$ RLF of FR II sources, adopting the translation evolution in the form used by Condon (1984), and

(ii) $\lambda[D(L, z)]$ - their Linear-size Function, assumed to be independent of the space density of sources, in the form:

$$
\lambda[D(L, z)]=\frac{(1 / 2)^{p}}{\Gamma(p)}\left(D / D_{f}\right)^{p-1} \exp \left(-D / 2 D_{f}\right)
$$

where a fiducial size is $D_{f}=D_{o}\left(L / L_{o}\right)^{m}(1+z)^{n}$. Giants of FR I-type are excluded from this analysis.

The RLF of FR II sources is determined by the best fit to the observed normalized source-counts at $1.4 \mathrm{GHz}$ (Figure 1), while the Linear-size Function - by the best fit to the distributions of apparent angular size and projected linear size of FR II sources, divided into two subsamples, with $z<0.5$ and $0.5 \leq z<5$ \{Figure $2(a, b)\}$. 


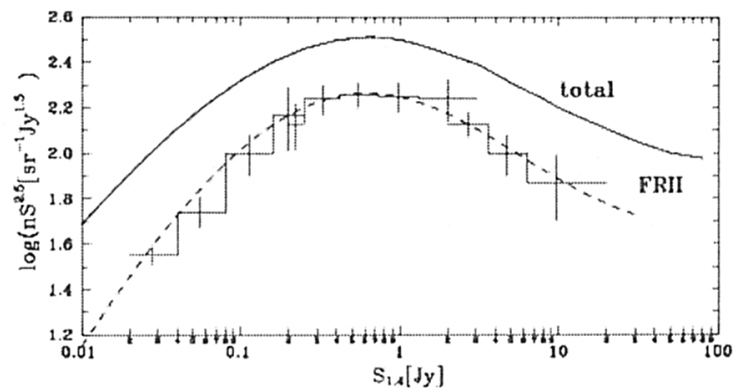

Figure 1. $\quad 1.4 \mathrm{GHz}$ normalized source counts of FR II sources
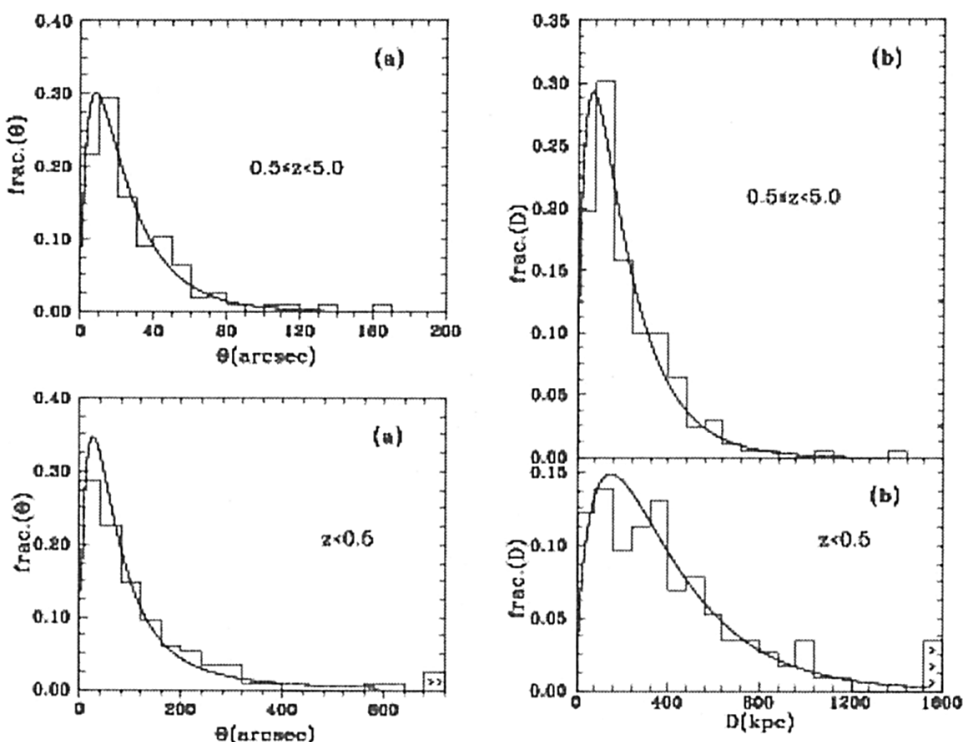

Figure 2. Linear-size function fitted to (a) angular size, and (b) projected linear size of FRII sources in two redshift ranges

\section{Results}

Having the function $\phi_{F R I I}(L, z, D)$, we calculated the total number of FR II giants as a function of $L, z$, and $D$. The resultant numbers of giants with $L_{1.4} \leq 10^{27.5} \mathrm{~W} \mathrm{~Hz}^{-1}$, expected from the model in the sky area of $8.2 \mathrm{sr}$ $\left(\left|b_{I I}\right|>20^{\circ}\right)$ and within three $1.4 \mathrm{GHz}$ flux-density ranges, are shown in Table 1. A comparison with the relevant observational data shows that the fraction of recognized giants gradually decreases with a drop of the total flux.

The distributions of known FR II giants in the $D-z$ diagram for sources with $S_{1.4} \geq 500 \mathrm{mJy}$, and $50 \mathrm{mJy}<S_{1.4} \leq 500 \mathrm{mJy}$, are shown in Figure $3(\mathrm{a}, \mathrm{b})$, respectively. Large full circles, crossed circles, and asterisks indicate giants with $10^{26.5}<L_{1.4} \leq 10^{27.5} \mathrm{~W} \mathrm{~Hz}^{-1}, 10^{25.5}<L_{1.4} \leq 10^{26.5} \mathrm{~W} \mathrm{~Hz}^{-1}$, and $L_{1.4} \leq 10^{25.5}$ $\mathrm{W} \mathrm{Hz}{ }^{-1}$, respectively. The $50 \%, 90 \%$, and $99 \%$ of the total number of giants, expected from the model in these three ranges of $1.4 \mathrm{GHz}$ luminosity, are bounded 
Table 1. Observed and expected numbers of FR II giants with $L_{1.4} \leq$ $10^{27.5} \mathrm{~W} \mathrm{~Hz}^{-1}$

\begin{tabular}{lccc}
\hline & $S_{1.4} \geq 2 \mathrm{Jy}$ & $500 \mathrm{mJy} \leq S<2 \mathrm{Jy}$ & $50 \mathrm{mJy} \leq S<500 \mathrm{mJy}$ \\
\hline observed & 9 & 26 & 11 \\
expected & 8.8 & 45.7 & 350 \\
\hline obs/expect. & 1.02 & 0.57 &
\end{tabular}

by the solid, large-dash, and short-dash curves, respectively . The expected and observed fractions of giants stronger than $500 \mathrm{mJy}$ are also compared in Table 2 .

Table 2. Expected and observed fractions of giants $\left(\mathrm{S}_{1.4}>500 \mathrm{mJy}\right)$

\begin{tabular}{llll}
\hline $\begin{array}{l}\text { expected } \\
\text { from the model }\end{array}$ & $\begin{array}{l}\text { observed with } \\
\leq 25.5\end{array}$ & $\begin{array}{l}\log L_{1.4} G H z \\
\leq 26.5\end{array}$ & $\begin{array}{l}{\left[\mathrm{W} \mathrm{Hz}^{-1}\right]} \\
\leq 27.5\end{array}$ \\
\hline $50 \%$ & $50 \% \pm 18 \%$ & $42 \% \pm 9 \%$ & $38 \% \pm 8 \%$ \\
$90 \%$ & $88 \% \pm 11 \%$ & $68 \% \pm 8 \%$ & $65 \% \pm 8 \%$ \\
$99 \%$ & $100 \%$ & $97 \% \pm 3 \%$ & $93 \% \pm 4 \%$
\end{tabular}

Although these fractions do agree perfectly in the weakest luminosity range, the observed fractions are smaller than the expected ones when the luminosity range is enlarged. This can suggest that the linear size of giants does not obey the redshift-dependent Size Function fitted to the sizes of typical FR II sources. Nevertheless, the consistence between the predictions and the available data is quite good for giants with $S>500 \mathrm{mJy}$. This is not the case for the second flux-density range. All 11 giants fainter than 500 mJy have $z>0.35,7$ of which were found in the 7C sample (cf. Cotter et al. 1996, MNRAS, 281, 1081; Lacy et al. 1999, ap-ph/9905358). There is no source in the $D-z$ plane, where most of them should be located according to the model predictions. Why are they absent?

\section{Missing Giants}

Inspecting the NVSS and FIRST maps (Condon et al. 1998, AJ, 115, 1693, and Becker et al. 1995, ApJ, 450, 559, respectively), we found numerous doubles consisting of low-brightness lobes separated by a few arc min, but without a radio core detected in the FIRST survey - thus difficult to identify with an optical galaxy. Usually the relevant optical field does not contain objects brighter than 19 - $19.5 \mathrm{mag}$, thus suggesting a rather distant host galaxy. On the other hand, a low radio luminosity estimated from the apparent radio surface-brightness (Machalski \& Jamrozy, in prep.), may implicate that (i) most of these giant candidates should be at redshifts $z<0.4$, and (ii) their host galaxies might have very low optical luminosity, not obeying the Hubble diagram for strong radio galaxies (e.g. Eales 1985, MNRAS, 213, 899). The latter effect was also suggested by Schoenmakers et al. (1998, in "The Young Universe", ASP Conf. Ser. Vol.146, p.84). 

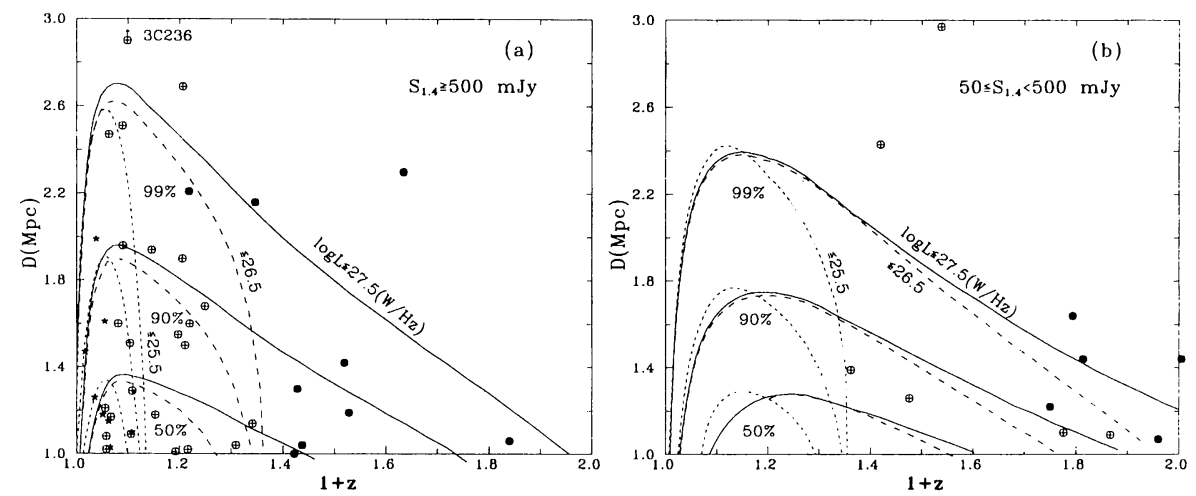

Figure 3. (a) Expected and observed D vs. $(1+\mathrm{z})$ distributions of giants with $S \geq 500 \mathrm{mJy}$. $50 \%, 90 \%$ and $99 \%$ of sources (each fraction up to three luminosity limits) are expected to lie in the areas under the respective curves (cf. the text). (b) the same, but for $50 \leq S<500$ mJy.

\section{Conclusions}

1) There is a tail of the largest linear extent of FR II sources which does not conform to any reasonable statistical distribution (here a $\Gamma$ distribution).

2) It is very likely that there are numerous unrecognized giant double sources of $1.4 \mathrm{GHz}$ luminosity $<10^{26.0-25.5} \mathrm{~W} \mathrm{~Hz}^{-1}$ at redshifts $<0.4$.

3) Many of them may be very old remnants of former activity and their parent galaxy may have been strongly evolved. This would contradict to the previous findings that the known, already well studied, giants with bright optical counterpart are not older than other doubles (e.g. Mack et al. 1998, A\&A, 329, 431; Cotter 1998, in "Observational Cosmology with the New Radio Surveys", Kluwer Acad. Publ., p.233 ).

4) Further spectral-ageing studies of these candidates in the radio, as well as optical identification and the follow-up photometry and spectroscopy of identified parent galaxies - are necessary to prove the above.

Acknowledgments. This work was supported in part by the State Committee for Scientific Research (KBN) under contract PB 0266/PO3/99/17. 